\title{
Collapsing shells of radiation in anti-de Sitter spacetimes and the hoop and cosmic censorship conjectures gr-qc/9812078
}

\author{
José P. S. Lemos \\ Departamento de Astrofísica, Observatório Nacional-CNPq, Rua General José Cristino 77, 20921 Rio de Janeiro, Brazil E6 \\ Departamento de Física, Instituto Superior Técnico, Av. Rovisco Pais 1, 1096 Lisboa, Portugal.
}

\begin{abstract}
Gravitational collapse of radiation in an anti-de Sitter background is studied. For the spherical case, the collapse proceeds in much the same way as in the Minkowski background, i.e., massless naked singularities may form for a highly inhomogeneous collapse, violating the cosmic censorship, but not the hoop conjecture. The toroidal, cylindrical and planar collapses can be treated together. In these cases no naked singularity ever forms, in accordance with the cosmic censorship. However, since the collapse proceeds to form toroidal, cylindrical or planar black holes, the hoop conjecture in an anti-de Sitter spacetime is violated.
\end{abstract}

PACS numbers: 04.20.Jb, 97.60.Lf.

\section{Introduction}

The cosmic censorship conjecture [1] forbids the existence of naked singularities, singularities not surrounded by an event horizon. The hoop conjecture [2] states that BHs form when and only when a mass $M$ gets compacted into a region whose circumference in every direction is less than its Schwarzschild circumference $4 \pi M(G=c=1)$. The collapse of spherical matter in the form of dust, or radiation forms massless shell-focusing naked singularities violating the cosmic censorship 33 10].

It is also violated for matter with cylindrical symmetry where the collapse proceeds to form a line singularity [11]. The hoop conjecture has not suffered from such counter-examples. Cylinders, for instance, do not get compacted, and do not form black holes. Spindles form black holes only when all collapsing directions are sufficiently compactified in accord with the hoop conjecture [12]. These results follow from analysis in asymptotically flat spacetimes.

In this work we want to study how these features might get modified in an anti-de Sitter background. We study imploding radiation in an anti-de Sitter background both in spherically symmetric and toroidally, cylindrical or plane symmetric spaces, to test the appearance of naked singularities against the cosmic censorship conjecture and the formation of toroidal, cylindrical or planar black holes against the hoop conjecture.

The Vaidya metric, describing a spherically symmetric spacetime with radiation, is an exact solution of Einstein's field equations which has been used to generate shell-focusing naked singularities. Its straightforward generalization to spherically symmetric spacetimes with negative cosmological constant is called the Vaidya-anti-de Sitter metric. In addition, to describe collapsing radiation in spacetimes with toroidal, cylindrical or planar symmetry and a negative cosmological constant, there is an appropriately modified Vaidya metric, which of course, satisfies Einstein's field equations.

The spherically symmetric case with zero cosmological constant has been thoroughly studied (see [13] for a review). It is usually admitted that in spherical symmetry the effects of adding a negative cosmological constant $\Lambda$ do not alter radically the description. The main difference is that the exterior spacetime should be described by the Vaidyaanti-de Sitter metric. As we shall see the situation can change drastically for a collapse with non-spherical topology. Indeed, we find that (i) in spherical symmetry with negative $\Lambda$, massless naked singularities form for a sufficiently inhomogeneous collapse, similarly to the $\Lambda=0$ case and (ii) toroidal, cylindrical or plane symmetric collapse with negative $\Lambda$ does not produce naked singularities; instead black holes will form giving an explicit counter-example to the hoop conjecture.

\section{Spherical Collapse}

The Einstein field equations are

$$
G_{a b}+\Lambda g_{a b}=8 \pi T_{a b},
$$

where $G_{a b}, g_{a b}, T_{a b}$ are the Einstein, the metric and the the energy-momentum tensors, respectively, and $\Lambda$ is the cosmological constant $(G=c=1)$. In the spherically symmetric case the equations admit the Vaidya solution

$$
\begin{gathered}
d s^{2}=-\left(1+\alpha^{2} r^{2}-\frac{2 m(v)}{r}\right) d v^{2}+2 d v d r+ \\
+r^{2}\left(d \theta^{2}+\sin ^{2} \theta d \phi^{2}\right)
\end{gathered}
$$


for an energy-momentum tensor given by

$$
\begin{aligned}
T_{a b} & =\frac{1}{4 \pi r^{2}} \frac{d m(v)}{d v} k_{a} k_{b} \\
k_{a} & =-\delta_{a}^{v}, \quad k_{a} k^{a}=0 .
\end{aligned}
$$

Here $\alpha \equiv \sqrt{\frac{-\Lambda}{3}}, v$ is a null coordinate, called the advanced time, with $-\infty<v<\infty, r$ is the radial coordinate with $0<r<\infty$, and $\theta, \phi$ are the coordinates which describe the two-dimensional spherical surface. The Vaidya metric (2) describes the gravitational field of a spherical flow of unpolarized radiation in the geometrical optics approximation. It represents a spherical null fluid. Noting that the energy-density of the radiation is $\epsilon=\frac{2}{4 \pi r^{2}} \frac{d m}{d v}$, one sees that the weak energy condition for the radiation is satisfied whenever $\frac{d m}{d v} \geq 0$, i.e., the radiation is imploding. The function $m(v)$ represents a mass and is thus a non-negative increasing function of $v$.

The physical situation we want to represent is of radiation injected with the velocity of light radially into an anti-de Sitter spacetime from infinity towards the center. For $v<0$ the spacetime is anti-de Sitter with $m(v)=0$. At $v=V$, say, the radiation is turned off. For $v>V$ the exterior spacetime settles into a Schwarzschild-anti-de Sitter spacetime. The first spherical ray arrives at the center when $r=0$ and $v=0$, forming a singularity. We will now test whether future directed null geodesics terminate at the singularity. If they do, the singularity is naked.

In these coordinates, lines with $v=$ constant represent incoming radial null vectors whose generator vectors have the form $k^{a}=(0,-1,0,0)$, with $k_{a}=(-1,0,0,0)$ (see equation (3)). The generators $l^{a}$ of outgoing null lines are then given by $l^{a}=\left(1, \frac{1}{2}\left(1+\alpha^{2} r^{2}-\frac{2 m(v)}{r}\right), 0,0\right)$ with, $l_{a} l^{a}=0$ and $l_{a} k^{a}=-1$. The equation for outgoing radial null geodesics $v(r)$ is then

$$
\frac{d v}{d r}=\frac{l^{v}}{l^{r}}=\frac{2 r}{r+\alpha^{2} r^{3}-2 m(v)} .
$$

Equation (4), for future null geodesics, has a singular point at $r=0$ and $v=0$. The nature of the singular point can now be analysed. Following [14] we write

$$
2 m(v)=\lambda v+f(v) .
$$

where $\lambda$ is a constant and $f(v)=o(v)$ as $v \rightarrow 0$. Following standard techniques [15] one can see that the singularity is a unstable node when $0 \leq \lambda \leq \frac{1}{8}$. Since $\mu \equiv 1 / \lambda$ gives a degree of inhomogeniety of the collapse (see 10]), we see that for a collapse sufficiently inhomogeneous naked singularities develop. This result is the same result as in collapsing radiation in a Minkowski background, as it should have been expected, since when $r \rightarrow 0$ the cosmological constant term $\alpha^{2} r^{2}$ is negligible. The global nakedness of the singularity can then be seen by making a junction onto the Schwarzschild-anti-de Sitter spacetime.

The Kretschmann scalar is given by $K=R^{a b c d} R_{a b c d}=a \frac{\lambda^{2}}{r^{4}}$ for some number $a$. Thus as $r \rightarrow 0$ the collapse forms a polynomial curvature singularity. To see if, in addition, the naked singularity is strong we examine the scalar $\psi \equiv \lim _{l \rightarrow 0} l^{2} G_{a b} l^{a} l^{b}$, where $l^{a}$ is the null geodesic along the Cauchy horizon parametrized by $l$. This gives $\psi=4 \lambda$, showing that the singularity is strong.

In 116 another solution for outgoing light rays is presented. This solution is also valid here for sufficiently small radii. Indeed, it is found that if $2 m(v)=\beta v^{\sigma}\left(1-2 \sigma \beta v^{\sigma-1}\right)$, with $\sigma>1$ and $\beta>0$ constants, then light rays with $r=v^{\sigma}$ emanate from a strong naked singularity.

Thus, for spherical symmetry, the cosmic censorship is violated for a sufficiently inhomogeneous collapse. On the other hand, the hoop conjecture is validated since a black hole forms whenever the spherical surface of matter passes its own gravitational radius.

\section{Toroidal, Cylindrical or Planar Collapse}

We now study the phenomenon of gravitational collapse of toroidal cylindrical or planar shells of radiation to verify whether naked singularities can occur.

Einstein's filed equations (1), have also the solution

$$
d s^{2}=-\left(\alpha^{2} r^{2}-\frac{q m(v)}{r}\right) d v^{2}+2 d v d r+r^{2}\left(d \theta^{2}+d \phi^{2}\right),
$$

for an energy-momentum tensor given by

$$
\begin{aligned}
T_{a b} & =\frac{q}{8 \pi r^{2}} \frac{d m(v)}{d v} k_{a} k_{b}, \\
k_{a} & =-\delta_{a}^{v}, \quad k_{a} k^{a}=0,
\end{aligned}
$$


where now, $\theta, \phi$ are the coordinates which describe the two-dimensional zero-curvature space generated by the twodimensional commutative Lie group $G_{2}$ of isometries. The topologies of this two-dimensional space can be (i) $T^{2}=$ $S^{1} \times S^{1}$, the flat torus model $\left[G_{2}=U(1) \times U(1)\right]$, (ii) $R \times S^{1}$, the cylindricallly symmetric model $\left[G_{2}=R \times U(1)\right]$, and (iii) $R^{2}$, the planar model $\left[G_{2}=E_{2}\right]$. In the toroidal case we choose $0 \leq \theta<2 \pi, 0 \leq \phi<2 \pi$, in the cylindrical case $-\infty<\theta<\infty, 0 \leq \phi<2 \pi$, and in the planar case $-\infty<\theta<\infty,-\infty<\phi<\infty$. The parameter $q$ has different values depending on the topology of the two-dimensional space. For the torus $q=\frac{2}{\pi}$ and $m(v)$ is a mass, for the cylinder $q=\frac{4}{\alpha}$ and $m(v)$ is a mass per unit length, and for the plane $q=\frac{2}{\alpha^{2}}$ and $m(v)$ is a mass per unit area. The values of the parameter $q$ given above, were taken from ADM masses of the corresponding static BHs found in 17 19. Setting $m(v)=$ const and $\alpha=0$ one obtains the Taub metric [20]. In this case, when $\alpha=0$, there are no black hole solutions [19.

As in the spherical symmetric case, metric (6) describes the gravitational field of a toroidal, cylindrical or planar flow of unpolarized radiation in the geometrical optics approximation. In the same way, for imploding radiation, $\frac{d m}{d v} \geq 0$, one has $\epsilon=\frac{q}{8 \pi r^{2}} \frac{d m}{d v}>0$, and the mass $m(v)$ is a non-negative increasing function of $v$.

One now injects radiation radially towards the center. If one whishes, one turns off the radiation at $v=V$, say, and then makes a junction with the exterior background spacetime, sometimes called Riemann-anti de Sitter spacetime [21. The first spherical ray arrives at the center for $r=0, v=0$, forming a singularity.

Lines with $v=$ constant represent incoming radial null geodesics, with generators $k^{a}=(0,-1,0,0)$, The generators $l^{a}$ of outgoing null lines are $l^{a}=\left(1, \frac{1}{2}\left(\alpha^{2} r^{2}-\frac{q m(v)}{r}\right), 0,0\right)$. The equation for outgoing radial null geodesics $v(r)$ is now

$$
\frac{d v}{d r}=\frac{l^{v}}{l^{r}}=\frac{2 r}{\alpha^{2} r^{3}-q m(v)} .
$$

Again, equation (8) for future null geodesics has a singular point at $r=0$ and $v=0$, and we write as in (5)

$$
q m(v)=\lambda v+\mathrm{o}(v)
$$

Following standard techniques one finds that the singularity is a center for all $\lambda$, and no radial future null geodesics terminate at the singularity. This is in contrast with the spherically symmetric collapse. In turn the collapse proceeds to form toroidal, cylindrical or planar black holes, once the exterior event horizon condition is achieved, i.e., $r=$ $\left(\frac{q M}{\alpha^{2}}\right)^{1 / 3}$, where $M$ is the total mass of the collapsing radiation, (see also 22]).

One can also try the Joshi and Dwivedi's type solutions [16]. However these give for the mass $q m(v)=\beta \alpha^{2} v^{3}-2 \beta^{\frac{2}{3}} v$, for some arbitrary constant $\beta$. They are unphysical since $\left.\frac{a m}{d v}\right|_{v=0}<0$.

Thus, for toroidal, cylindrical or planar collapse of radiation, naked singularities do not form, in accord with the cosmic censorship conjecture. On the other hand, cylindrical black holes as well as toroidal and planar ones do form, giving an explicit example of violation of the hoop conjecture.

Note that these conclusions are valid as long as $\alpha>0$. For $\alpha=0$ the collapse proceeds to form a naked singularity with toroidal, cylindrical, or planar symmetry and the Taub solution is the final background spacetime. In such case the cosmic censorship is violated, whereas the hoop conjecture is not [2,11].

\section{Conclusions}

The Vaidya metric has been extensively used to study the formation of naked singularities in spherical gravitational collapse 13. We have extended this study here to include a negative cosmological constant, and found that locally, in the vicinity of the singularity, the same results prevail. On the other hand, we have found that collapse of toroidal, cylindrical or planar radiation in a spacetime with negative cosmological constant does not yield naked singularities, but always black holes. This shows that non-spherical collapse in a negative cosmological constant background may violate the hoop but not the cosmic censorship conjecture. 
[1] R. Penrose, Riv. Nuovo Cimento 1 (Numero Special), 252 (1969).

[2] K. S. Thorne, in Magic without Magic, ed. J. R. Klauder, Freeman and Company (1972), p. 231.

[3] D.M. Eardley and L. Smarr, Phys. Rev. D 19, 2239 (1979).

[4] D. Christodoulou, Comm. Math. Phys. 93, 171 (1984).

[5] A. Ori and T. Piran, Phys. Rev. Lett. 59, 2137 (1987).

[6] A. Papapetrou, in A Random Walk in Relativity and Gravitation, edited by N. Dadich, J.K. Rao, J.V. Narlikar and C.V. Vishveshwara (Wiley, New York, 1985).

[7] W. A. Hiscock, L. G. Williams, D. M. Eardley, Phys. Rev. D 26, 751 (1982).

[8] I. H. Dwivedi, P. S. Joshi, Class. Quantum Grav. 8, 1339 (1991).

[9] K. Lake, Phys. Rev. Lett. 68, 3129 (1992).

[10] J. P. S. Lemos, Phys. Rev. Lett. 68, 1447, (1992).

[11] T. A. Apostolatos, K. S. Thorne, Phys. Rev. D 46, 2435 (1992).

[12] S. L. Shapiro, S. A. Teukolsky, Phys. Rev. Lett. 66, 944 (1991).

[13] P. S. Joshi, Global Aspects in Gravitation and Cosmology, (Clarendon Press, Oxford, 1993).

[14] K. Lake, Phys. Rev. D 43, 1416 (1991).

[15] D. W. Jordan, P. Smith, Nonlinear Ordinary Differential Equations, (Clarendon Press, Oxford, 1987).

[16] P. S. Joshi, I. H. Dwivedi, J. Math. Phys. 32, 2167 (1991).

[17] J. P. S. Lemos, V. T. Zanchin, Phys. Rev. D 54, 3840 (1996).

[18] J. P. S. Lemos, Phys. Lett. B 352, 46 (1995).

[19] J. P. S. Lemos, Class. Quantum Gravity 12, 1081 (1995).

[20] A. Taub, Phys. Rev. 103, 454 (1956).

[21] L. Vanzo, Phys. Rev. D 56, 6475 (1997).

[22] J. P. S. Lemos, Phys. Rev. D 57, 4600 (1998). 